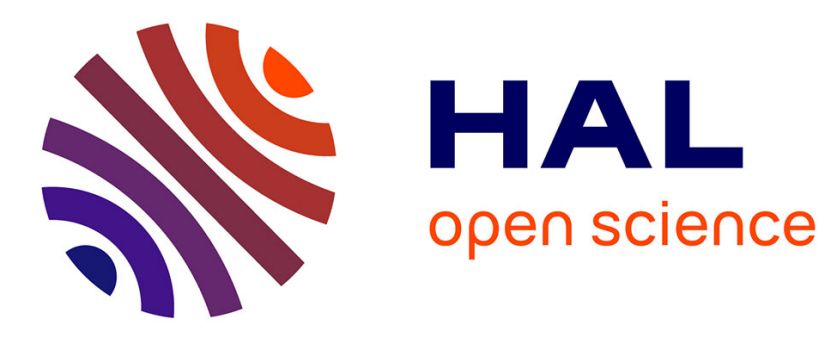

\title{
Propriétés électriques d'hétérostructures a-GaAs/c-GaAs(n) et de structures de type MIS a-GaAsN/c-GaAs(n)
}

Khalifa Aguir, A. Fennouh, H. Carchano, D. Lollman

\section{To cite this version:}

Khalifa Aguir, A. Fennouh, H. Carchano, D. Lollman. Propriétés électriques d'hétérostructures aGaAs/c-GaAs(n) et de structures de type MIS a-GaAsN/c-GaAs(n). Journal de Physique III, 1995, 5 (10), pp.1573-1585. 10.1051/jp3:1995211 . jpa-00249402

\section{HAL Id: jpa-00249402 https://hal.science/jpa-00249402}

Submitted on 1 Jan 1995

HAL is a multi-disciplinary open access archive for the deposit and dissemination of scientific research documents, whether they are published or not. The documents may come from teaching and research institutions in France or abroad, or from public or private research centers.
L'archive ouverte pluridisciplinaire HAL, est destinée au dépôt et à la diffusion de documents scientifiques de niveau recherche, publiés ou non, émanant des établissements d'enseignement et de recherche français ou étrangers, des laboratoires publics ou privés. 


\title{
Propriétés électriques d'hétérostructures a-GaAs/c-GaAs(n) et de structures de type MIS a-GaAsN/c-GaAs(n)
}

\author{
K. Aguir, A. Fennouh, H. Carchano et D. Lollman \\ Laboratoire d'électronique et Physico-chimie des Couches Minces, EA885 Faculté des Sciences \\ et Techniques de St-Jérôme, Case A62, 13397 Marseille cedex 20, France
}

(Reçu le 23 décembre 1994, révisé le 13 mars 1995 et le 28 juin 1995, accepté le 6 juillet 1995)

\begin{abstract}
Résumé. - L'étude porte sur des couches minces de GaAs et de GaAsN amorphes déposées par pulvérisation cathodique RF réactive sur des substrats de GaAs cristallin. Les caractéristiques électriques $I(V)$ et $C(V)$ ont été mesurées. Les hétérojonctions a-GaAs/c-GaAs(n) présentent un effet redresseur. Cet effet laisse place à une caractéristique symétrique avec une forte atténuation de l'intensité du courant pour les structures a-GaAsN/c-GaAs(n). Les structures réalisées ont alors un comportement semblable à celui d'une structure MIS imparfaite. L'existence d'une charge positive fixe dans le a-GaAsN a été mise en évidence. La densité des états d'interface au milieu de la bande interdite est évaluée à quelques $10^{11} \mathrm{~cm}^{-2} \mathrm{eV}^{-1}$
\end{abstract}

\begin{abstract}
Heterojunctions were fabricated by deposit of amorphous GaAs and GaAsN on c-GaAs. $I(V)$ and $C(V)$ measurements were performed to determine electrical properties of these structures. The a-GaAs/c-GaAs(n) heterojunctions present a p-n junction like behaviour. The characteristics of the a-GaAsN/c-GaAs(n) heterojunctions present a MIS like structure behaviour with some imperfections. A fixed positive charge was detected and a density of interface states of about $10^{11} \mathrm{eV}^{-1} \mathrm{~cm}^{-2}$ was evaluated.
\end{abstract}

\section{Introduction}

Les semiconducteurs amorphes furent assez peu étudiés jusqu'en 1970, année où Chittik [1] signale la possibilité de doper le silicium amorphe (a-Si) obtenu par décomposition de silane dans une décharge luminescente. Ensuite, entre 1975 et 1979, Spear et Le Comber [2-4] réalisent de nombreux travaux sur ce matériau et montrent en particulier le rôle prépondérant de l'hydrogène dans la compensation des défauts du a-Si. Cet effet semble s'appliquer à d'autres semiconducteurs du groupe IV comme le germanium amorphe [3].

Alors que les travaux sur le silicium amorphe connurent un développement spectaculaire, avec notamment des applications photovoltaïques, les écrans plats ou les transistors en films minces (TFT), les études sur le GaAs amorphe (a-GaAs) furent très peu nombreuses. Pourtant la comparaison des propriétés optoélectroniques du Si et du GaAs cristallins permet d'espérer 
un comportement plus avantageux du a-GaAs en particulier dans les domaines photovoltaïque et optoélectronique.

Dès 1972, Beyer et Stuke [5] étudièrent les phénomènes de transport dans des couches minces a-GaAs déposées par évaporation flash. En 1977, Hauser [6] trouve par RPE une densité d'états localisée de l'ordre de $10^{19} \mathrm{eV}^{-1} \mathrm{~cm}^{-3}$. La même année Paul [7] rapporta l'influence de l'hydrogène sur les propriétés électriques et optiques du a-GaAs et suppose que l'hydrogène a un effet analogue à celui observé sur le silicium.

En France, ce semiconducteur fut particulièrement étudié par trois laboratoires.

Theye et Gheorghiu [8-15] étudièrent $\mathrm{GaAs}, \mathrm{GaP}$ et $\mathrm{GaSb}$ amorphes déposés par évaporation flash. Leur contribution porte particulièrement sur la caractérisation optique en corrélation avec le désordre du matériau et différents traitements : recuit, post-hydrogénation.

Aguir et Despax étudièrent les composés a- $\mathrm{Ga}_{x} \mathrm{As}_{1-x} \mathrm{H}$ obtenus par pulvérisation réactive R.F. d'une cible de gallium et décomposition d'arsine [16-19].

Notre travail a démarré en 1979 par l'étude de a-GaAs obtenu par pulvérisation cathodique R.F. Cette étude comportait l'hydrogénation du matériau par pulvérisation réactive dans un plasma d'argon-hydrogène $[20,21]$. Elle a permis de déterminer les conditions expérimentales permettant d'obtenir un matériau stœchiométrique et amorphe [22-25]. L'étude des caractéristiques électriques et physico-chimiques de ce matériau $[26,27]$ a montré que le a-GaAs déposé à température ambiante contient un excès d'arsenic et a une résistivité de l'ordre de $10^{5} \Omega \mathrm{cm}$ avec un gap optique de $1,1 \mathrm{eV}$. L'hydrogénation élargit ce gap jusqu'à $1,45 \mathrm{eV}$ et la résistivité augmente de quatre décades environ. L'élévation de la température du substrat permet d'obtenir un matériau stœechiométrique vers $300^{\circ} \mathrm{C}$. Dans ce cas, la résistivité est de l'ordre de $10^{9} \Omega \mathrm{cm}$ et la largeur du gap optique est de $1,5 \mathrm{eV}$. La densité d'états localisés est alors de l'ordre de $4 \times 10^{16} \mathrm{eV}^{-1} \mathrm{~cm}^{-3}$

Cette étude s'est poursuivie par la réalisation d'hétérojonctions a-GaAs/c-Si obtenues sur silicium de type $\mathrm{p}$ [28] et sur silicium de type $\mathrm{n}$ [29]. Pour favoriser la croissance d'une phase amorphe, il est nécessaire de déposer la couche à faible tension d'autopolarisation (faible puissance R.F.) tout en gardant un produit pression-distance inter-électrodes élevé. Dans ces conditions, la température maximale de dépôt donnant un film amorphe est de $150^{\circ} \mathrm{C}$ et le matériau obtenu contient un léger excès d'arsenic (51\%). A la température ambiante les courants directs sont de l'ordre de $10^{-4} \mathrm{~A} / \mathrm{cm}^{2}$ pour une polarisation de $0,4 \mathrm{~V}$ sur $\operatorname{Si}(\mathrm{p})$ et de $0,7 \mathrm{~V}$ pour $\operatorname{Si}(\mathrm{n})$. Le processus de conduction dans ces structures pourrait être interprété par un processus de capture-émission par effet tunnel pour le courant direct alors que le courant inverse serait dû à un phénomène de génération.

Nous sommes ensuite passé à la réalisation d'hétérostructures a-GaAs/c-GaAs. L'objectif était d'étudier la possibilité de réaliser une structure MIS en utilisant le a-GaAs comme passivant. La réalisation d'une capacité MIS sur GaAs préoccupe depuis longtemps les chercheurs [33-44]. Les principaux inconvénients rencontrés sont soit des températures d'élaboration trop élevées $\left(>400^{\circ} \mathrm{C}\right)$, soit des désaccords de structure importants entre le diélectrique et le substrat. De ce fait, les structures obtenues comportent trop d'états d'interface $\left(\geq 10^{13} \mathrm{eV}^{-1} \mathrm{~cm}^{-2}\right)$ qui immobilisent le niveau de Fermi du c-GaAs.

Notre méthode d'élaboration permet de nous affranchir de ces deux inconvénients : le dépôt se fait à température peu élevée $\left(\leq 150^{\circ} \mathrm{C}\right)$ et, de plus, les propriétés physico-chimiques du a-GaAs sont pratiquement comparables à celles du substrat. On peut cependant noter l'insuffisance de la résistivité de a-GaAs $\left(10^{8}-10^{9} \Omega \mathrm{cm}\right)$ comparée à celle d'un bon diélectrique.

C'est la raison pour laquelle nous avons ensuite réalisé, par pulvérisation réactive dans un mélange d'argon et d'azote, des couches minces amorphes de GaAsN afin d'augmenter la résistivité des films déposés. 
Le travail que nous décrivons dans ce document porte sur les hétérostructures a-GaAs/c$\mathrm{GaAs}(\mathrm{n})$ et a-GaAsN/c-GaAs(n). Le dépôt de a-GaAs donne une hétérojonction alors que celui de GaAsN donne une structure de type MIS.

\section{Etude des structures a-GaAs/c-GaAs}

2.1. PrÉParation des ÉChantillons. - Les substrats utilisés sont constitués d'une couche de GaAs d'environ $3 \mu \mathrm{m}$ d'épaisseur épitaxiée sur des plaquettes de GaAs très fortement dọpées. La concentration des états donneurs dans les couches épitaxiées sont : $N_{\mathrm{D}}=9 \times 10^{15} \mathrm{~cm}^{-3}$ La surface des substrats utilisés est d'environ $0,6 \mathrm{~cm}^{2}$.

Le dépôt des couches de GaAs amorphe est fait par pulvérisation d'une cible monocristalline de GaAs dans un bâti de pulvérisation cathodique R.F. conventionnel. L'épaisseur des couches déposées est d'environ $500 \mathrm{~nm}$.

Les paramètres de dépôt ont été fixées à la suite d'une étude expérimentale approfondie sur les conditions de dépôt de films de GaAs amorphe sur des substrats de Si monocristallins [28-30]. Seule la température des substrats de GaAs a été maintenue à l'ambiante pour éviter la croissance de couches polycristallines qui présentent d'autres propriétés.

Les conditions expérimentales de dépôt utilisées lors de cette étude sont : pression d'argon de 6,67 Pa, distance inter-électrodes de $3 \mathrm{~cm}$, tension d'autopolarisation comprise entre 600 et 700 Volts ce qui correspond à une puissance radiofréquence de 25-30 Watts, durée de dépôt de 1,5 heures. Le substrat est à la température ambiante, car des températures plus élevées favorisent la croissance de couches cristallines. Le choix de ces conditions expérimentales a été dicté par l'étude sur le GaAs amorphe évoquée ci-dessus.

Le matériau amorphe ainsi obtenu a les caractéristiques d'un semiconducteur type $\mathbf{n}$ de résistivité élevée $\left(10^{5} \Omega \mathrm{cm}\right)$ avec une bande interdite de l'ordre de $1,1 \mathrm{eV}$ [27].

Après réalisation du dépôt, des contacts métalliques sont évaporés sur chacune des faces de l'échantillon. Sur les films de a-GaAs, les contacts sont en or. Ils ont une surface moyenne de $0,78 \times 10^{-2} \mathrm{~cm}^{2}$. Sur les substrats de GaAs-n ${ }^{+}$c'est l'indium qui est utilisé. Ces contacts sont décrits comme ohmiques dans la littérature [17,29].

Les caractéristiques $C-V$ des structures étudiées sont mesurées à l'aide d'un pont RLC HP $4275 \mathrm{~A}$ multi-fréquences. Les caractéristiques $I-V$ sont obtenues à l'aide d'un électromètre HP 4140B.

2.2. RÉSultats ET DisCussions. - Nous présentons sur la figure 1a) la structure de test utilisée pour les mesures électriques. La figure 1b) montre un exemple de caractéristique capacitétension à $1 \mathrm{MHz}$. Les caractéristiques courant-tension sont montrées sur les figures 2 et 3 .

Toutes ces caractéristiques ont été relevées à température ambiante. Leur examen permet de faire des constatations qualitatives communes aux deux types de structures étudiées :

- Une variation assez grande de la capacité est observée traduisant le développement d'une zone de charge d'espace importante au niveau de la jonction amorphe-cristal.

- La capacité continue d'augmenter quand la structure est polarisée en directe.

- Les courants directs et inverses, très dissymétriques, expriment un caractère redresseur marqué.

Dans les rares études concernant ce type de structure, Kumabe et al. [45] ont observé des caractéristiques semblables.

Les modèles d'hétérojonctions abruptes appliqués à ces structures a-GaAs/c-GaAs ne permettent pas de dégager les différents paramètres caractéristiques d'une hétérojonction comme les discontinuités des bandes, le potentiel de diffusion ou encore le diagramme énergétique. 


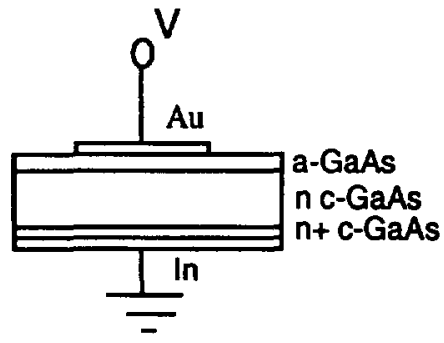

a)

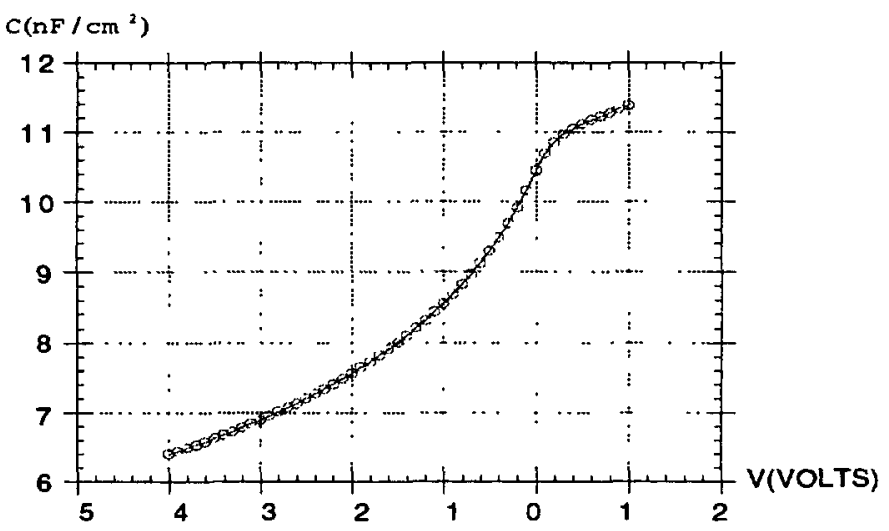

b)

Fig. 1. - a) Structure de test b) Caractéristique capacité-tension de la structure Au/a-GaAs/c$\operatorname{GaAs}(\mathbf{n}) / \mathrm{In}$.

[a) Test structure b) Capacitance-Voltage characteristics of Au/a-GaAs/c-GaAs(n)/In.]

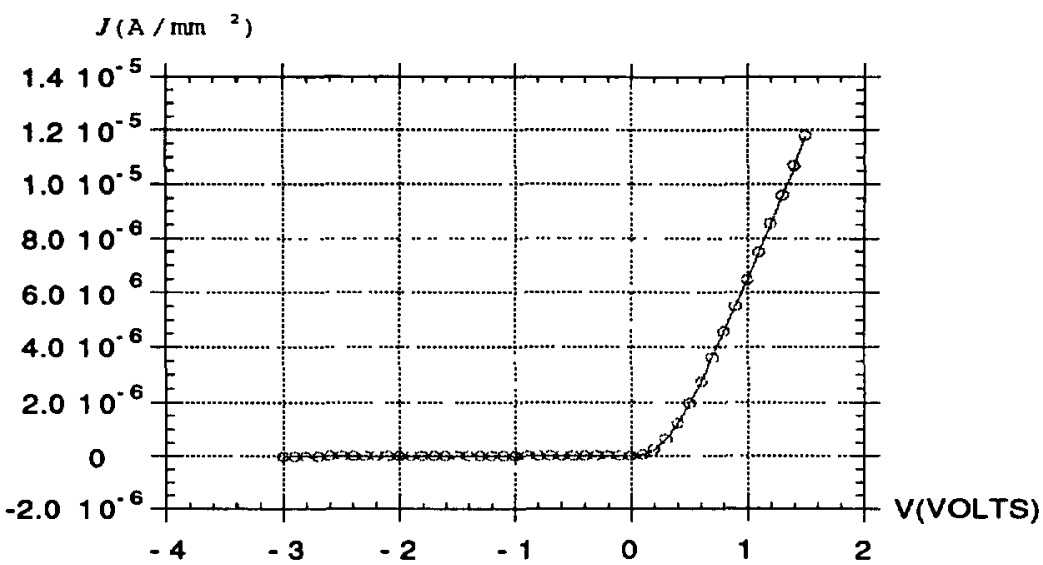

Fig. 2. - Caractéristique courant-tension de la structure Au/a-GaAs/c-GaAs(n)/In.

[Current density-Voltage characteristics of $\mathrm{Au} / \mathrm{a}-\mathrm{GaAs} / \mathrm{c}-\mathrm{GaAs}(\mathrm{n}) / \mathrm{In}$.]

En effet les caractéristiques $C^{-2}=f(V)$ ne sont pas linéaires ce qui rend très difficile leur exploitation même en utilisant les approximations que nous avons faites pour les structures a-GaAs/c-Si(p et $n$ ) $[28,29]$. Nous pensons qu'une importante densité d'état de surface est présente au niveau de l'interface. En effet, les films de a-GaAs sont déposés à froid ce qui entraîne une densité d'états élevée dans le gap [4]. De plus, la qualité de la surface des substrats est très sensible aux contaminations et à la formation d'oxydes. 


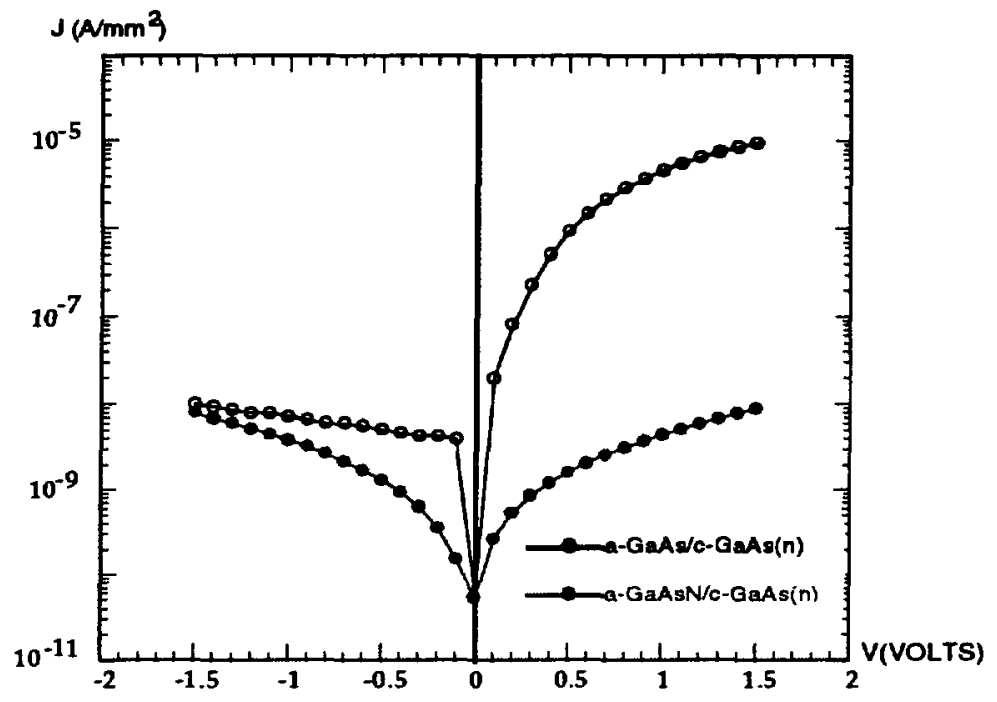

a)

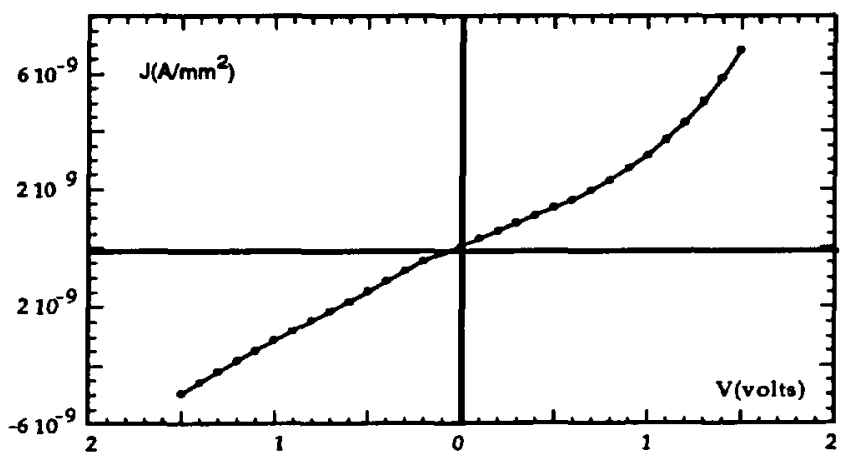

b)

Fig. 3. - Caractéristiques courant-tension des structures Au/a-GaAs/c-GaAs(n)/In a) et Au/aGaAsN/c-GaAs(n)/In a (log-lin), b) (lin-lin).

[Current density-Voltage characteristics of Au/a-GaAs/c-GaAs(n)/In a) and Au/a-GaAsN/c$\operatorname{GaAs}(\mathrm{n}) / \operatorname{In} \mathrm{a}(\log -\operatorname{lin}), \mathrm{b})(\operatorname{lin}-\operatorname{lin})$.

\section{Etude des structures a-GaAsN/c-GaAs (n)}

3.1. Le problème dANS la passivation de GaAs. - L'oxydation du GaAs engendre des surfaces instables et un isolant de qualité médiocre. Le digramme de phase du système Ga-As-O a été étudié en détail par Thurmond et al. [38]. Il montre la possibilité d'existence de diverses 
formes oxydées telles que $\mathrm{Ga}_{2} \mathrm{O}_{3}, \mathrm{As}_{2} \mathrm{O}_{3}, \mathrm{As}_{2} \mathrm{O}_{5}, \mathrm{GaAsO}_{4} \ldots$, et que les seules phases stables en présence de $\mathrm{GaAs}$ sont $\mathrm{Ga}_{2} \mathrm{O}_{3}, \mathrm{Ga}$ et $\mathrm{As}$. Ce résultat, bien qu'obtenu dans des conditions d'équilibre thermodynamique, semble pouvoir être étendu au cas des surfaces et interfaces de GaAs puisqu'il est bien établi que les oxydes $\mathrm{As}_{2} \mathrm{O}_{3}$ et $\mathrm{As}_{2} \mathrm{O}_{5}$ réagissent avec le GaAs en produisant de l'arsenic élémentaire à la surface du matériau. Les oxydes de GaAs ne se prêtent donc pas à une bonne passivation de la surface de ce matériau. Une des principales difficultés rencontrées dans la passivation de GaAs réside dans la nécessité de s'affranchir de la présence d'oxyde natif à sa surface et d'opérer dans une ambiance exempte d'oxygène [40]. Divers auteurs ont montré que la désoxydation-nitruration de GaAs suivie d'un dépôt de nitrure de silicium peut produire une bonne passivation de GaAs en particulier du point de vue de la morphologie de l'interface et de sa stabilité physico-chimique $[39,41]$.

Le GaN étant un isolant, nous avons alors fait l'hypothèse que le composé ternaire a-GaAsN aurait des propriétés d'isolant supérieures à celles du a-GaAs. S'il s'avérait de plus que si le composé GaAsN était stable, l'élaboration de structures MIS deviendrait possible.

Les alliages de GaAsN cristallins ont fait l'objet de très peu d'étude. Récemment Wayers et al. [46] publièrent un article sur l'élaboration par MOCVD de couches cristallines de $\mathrm{GaAs}_{1-x} \mathrm{~N}_{x}$ $(0<x<0,015)$. Quant aux alliages de GaAsN amorphes, aucune étude n'a été menée jusqu'à présent.

3.2. PRÉPARATION DES ÉCHANTILLONS. - Nous avons synthétisé par pulvérisation réactive des couches minces de a-GaAsN amorphe sur des substrats monocristallins de GaAs. Le gaz réactif, l'azote, est ajouté à l'argon en quantité contrôlée. La nitruration in-situ du dépôt a pu ainsi être effectuée.

Les conditions expérimentales utilisées pour l'élaboration des alliages de a-GaAsN sont celles décrites ci-dessus hormis la température de dépôt qui a été maintenue à $423 \mathrm{~K}$, la durée de dépôt à 30 minutes et le mélange d'argon et d'azote à $0,67 \mathrm{~Pa}$ d'azote pour une pression totale de 6,67 Pa. Ces paramètres ont été fixés (sans être optimisés) en fonction de travaux antérieurs sur le a-GaAs pour permettre une comparaison aisée des résultats en fonction de la présence d'azote.

Les résultats de spectroscopie RAMAN nous ont permis de vérifier le caractère amorphe des couches déposées. Les résultats d'analyse ESCA autorisent l'hypothèse d'une structure chimique de la forme a-GaAs $s_{1-x} \mathrm{~N}_{x}$. Les résultats publiés par Weyers [47] sur les alliages GaAsN confirment cette hypothèse. Cependant nous n'avons pu déterminer avec précision la composition de nos alliages.

3.3. RÉSUltats ET Discussions. - Les courbes qui illustrent la variation du courant qui traverse les structures a-GaAsN/c-GaAs(n) en fonction de la tension de polarisation à la température ambiante et à l'obscurité sont représentées dans un repère semi-logarithmique sur la figure $3 \mathrm{a}$ et en linéaire sur la figure $3 \mathrm{~b}$. Cette courbe est comparée à celle d'une structure formée avec du a-GaAs.

On peut tout d'abord constater une forte diminution de l'intensité du courant. Cette atténuation du courant confirme le caractère plus résistif des films de a-GaAsN. D'autre part, l'effet redresseur constaté sur les structures a-GaAs/c-GaAs a fait place à une caractéristique pratiquement symétrique. La figure $3 \mathrm{~b}$ montre que cette symétrie devient discutable Pour des polarisations directes supérieures à $0,8 \mathrm{~V}$. La non linéarité de la courbe pose alors le problème d'injection de charges à travers la couche de a-GaAsN qui doit probablement être à l'origine de l'augmentation du courant. 


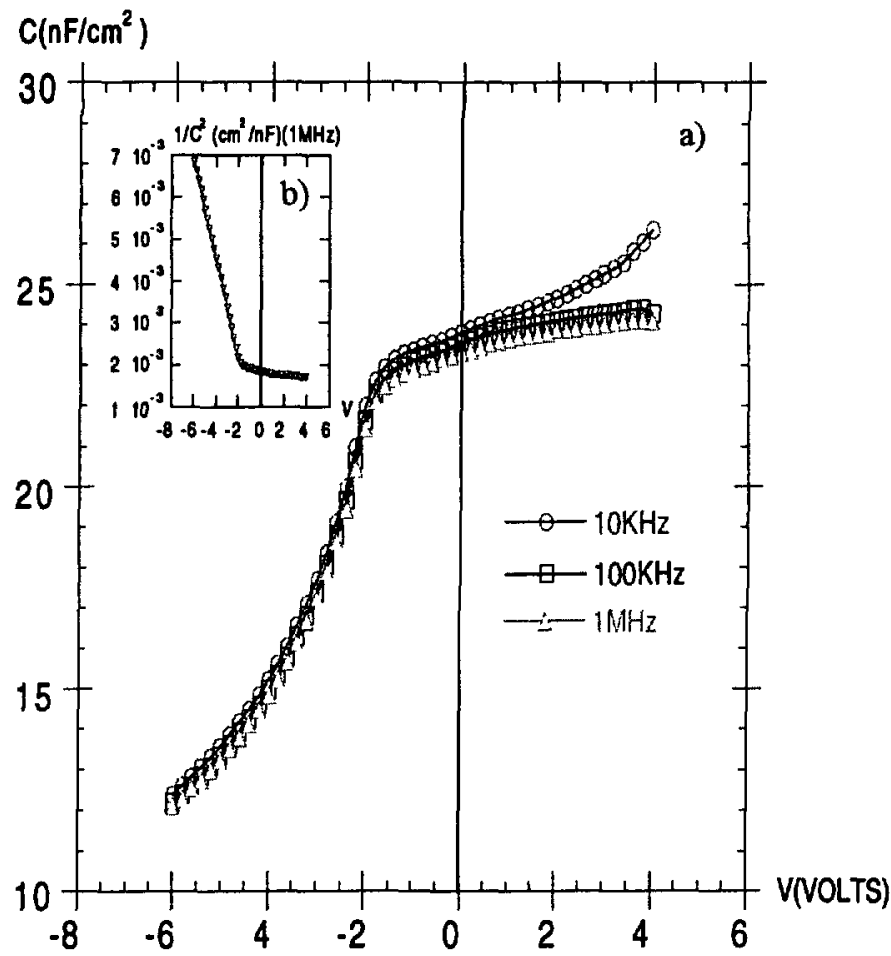

Fig. 4. - a) Caractéristique capacité-tension de la structure Au/a-GaAsN/c-GaAs(n)/In à $10 \mathrm{kHz}$, $100 \mathrm{kHz}$ et $1 \mathrm{MHz}$ b) $1 / \mathrm{C} 2$ en fonction de la tension.

(a) Capacitance-Voltage characteristics of $\mathrm{Au} / \mathrm{a}-\mathrm{GaAsN} / \mathrm{c}-\mathrm{GaAs}(\mathrm{n}) / \mathrm{In}$ at $10 \mathrm{kHz}, 100 \mathrm{kHz} 1 \mathrm{MHz}$ b) $1 / C^{2}$ versus voltage.]

Les courbes $C-V$ à trois fréquences et à température ambiante, des structures a-GaAsN/c$\operatorname{GaAs}(n)$ sont représentées sur la figure 4. Elles sont pratiquement confondues pour les différentes fréquences entre $10 \mathrm{kHz}$ et $1 \mathrm{MHz}$ et correspondent à celles que l'on peut obtenir avec une structure MIS présentant un certain nombre d'imperfections.

Le régime d'inversion n'est pas atteint alors qu'une amorce d'accumulation est observée. La capacité de ces structures continue à décroître pour des tensions inverses plus élevées et on peut supposer que les trous minoritaires, de concentration très faible, générés dans la zone de déplétion ne forment pas une couche d'inversion. Ils peuvent aussi s'échapper à travers les films de a-GaAsN. Un régime de déplétion profonde est donc observé. Ce régime reste stable, ce qui pose le problème de la mise en évidence de la génération des minoritaires. Quand au régime d'accumulation, son étude doit prendre en compte les pièges et l'injection de charges dans la couche de a-GaAsN. L'hypothèse d'une grande densité de pièges à l'interface qui bloquerait l'évolution des courbes $C-V$ pour certaines fréquences (cf. Fig. 4a) peut bien sûr être évoquée. Notons cependant qu'une mesure de la capacité d'une couche de a-GaAsN déposée sur c$\mathrm{Si}\left(\mathrm{n}^{+}\right)$donne une valeur constante et proche de $24 \mathrm{nF} / \mathrm{cm}^{2}$ dans les mêmes conditions que précédemment. La vérification de ces mesures passe par la connaissance de la valeur de la constante diélectrique de la couche de a-GaAsN. 
Les concentrations de dopants dans les substrats peuvent être calculées à partir de la capacité mesurée en déplétion [48] à la fréquence de $1 \mathrm{MHz}$, en utilisant l'équation :

$$
N_{A, \mathrm{D}}=2\left(\frac{\mathrm{d}\left(A / C_{\mathrm{m}}\right)^{2}}{\mathrm{~d} V}\right)^{-1}
$$

où $C_{\mathrm{m}}$ est la capacité mesurée, $A$ sa surface et $\varepsilon_{s}$ la constante diélectrique du GaAs.

Nous obtenons une valeur de $N_{\mathrm{D}}=9,4 \times 10^{15} \mathrm{~cm}^{-3}$ (voir Fig. $4 \mathrm{~b}$ ). Cette valeur est très proche de celle indiquée par le fournisseur.

Nous allons maintenant effectuer une étude comparative des caractéristiques $C(V)$ mesurées avec les caractéristiques $C(V)$ théoriques.

Le calcul de la caractéristique $C(V)$ d'une capacité MIS idéale nécessite la connaissance de la valeur de la capacité géométrique de l'isolant. Nous avons utilisé dans nos calculs la valeur asymptotique de la capacité à $1 \mathrm{MHz}$ en régime d'accumulation (Fig. 4).

Nous avons représenté sur la figure 5 , la courbe expérimentale et les courbes théoriques obtenues à partir de l'approximation de désertion [49] et du calcul exacte d'une capacité MIS [50].

La courbe $C(V)$ expérimentale suit parfaitement la courbe idéale en régime d'accumulation et en régime de déplétion lorsqu'on la translate d'une valeur constante le long de l'axe des tensions. Ce décalage mesuré à la tension de bandes plates $V_{\mathrm{FB}}$, qui correspond à une polarisation nulle dans le cas de la courbe idéale, peut être relié d'une part, à la différence des travaux de sortie du métal et du substrat semiconducteur $\phi_{\mathrm{ms}}$ et d'autre part, à la présence d'une charge fixe $Q$ dans l'isolant et dont le signe commande le sens du décalage.

Dans le cas de la figure 5 , la tension de bandes plates est de $-2 \mathrm{~V}$. La charge comprise dans l'isolant est donc positive et sa valeur peut être estimée à partir de la relation [51] :

où

$$
V_{\mathrm{FB}}=\phi_{\mathrm{ms}}-\frac{Q}{C_{\imath}}
$$

$$
\phi_{\mathrm{ms}}=\phi_{\mathrm{m}}-\left(\chi+\frac{E_{\mathrm{g}}}{2 q}-\psi_{\mathrm{B}}\right)
$$

représente la différence des travaux de sortie entre le métal et le semiconducteur avec: $\phi_{\mathrm{m}}=$ $4,8 \mathrm{eV}$ le travail de sortie du métal, $\chi=4,07 \mathrm{eV}$ l'affinité électronique du $\mathrm{GaAs}, E_{\mathrm{g}}=1,43$ $\mathrm{eV}$ la largeur de sa bande interdite et $\psi_{\mathrm{B}}=0,57 \mathrm{eV}$ l'écart en énergie entre le niveau de Fermi $E_{\mathrm{F}}$ et le niveau de Fermi intrinsèque $E_{\mathrm{i}}$ dans le volume.

$C_{\imath}=24 \mathrm{nF} / \mathrm{cm}^{2}$ est la capacité par unité de surface du diélectrique (a-GaAsN).

Nous obtenons $Q=3,9 \times 10^{11} \mathrm{~cm}^{-2}$

A partir des courbes de la figure 5 , nous pouvons faire une évaluation de la répartition énergétique de la densité des états d'interface par la méthode de Terman [52].

Cette méthode est simple et rapide. Mais elle est relativement imprécise et son utilisation est limitée aux structures présentant des fortes densités d'états d'interface ( $\left.>\mathrm{qq} 10^{11} \mathrm{~cm}^{-2} \mathrm{eV}^{-1}\right)$. En effet, pour des densités inférieures, l'écart entre les pentes des caractéristiques $C(V)$ théoriques et expérimentales est trop faible pour que cette méthode puisse être appliquée de façon fiable.

L'analyse Terman requiert d'autre part une connaissance précise du dopage du semiconducteur au voisinage de la surface. En pratique il est souvent obtenu à partir de courbes $C(V)$ en forte déplétion. Une autre source possible d'erreurs provient du choix de la fréquence de 


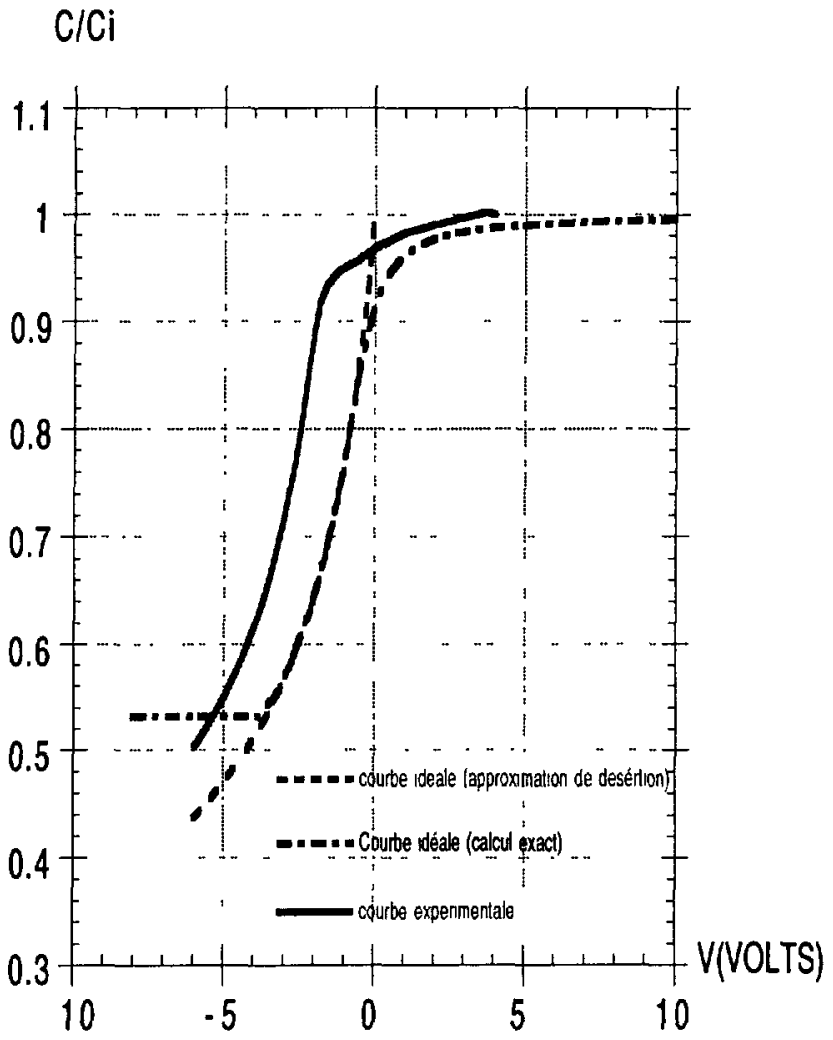

Fig. 5. - Caractéristiques capacité-tension théoriques et expérimentale de la structure a-GaAsN/cGaAs(n).

[Theoretical and experimental characteristics of a-GaAsN/c-GaAs(n) structure.]

mesure. Il apparaît en effet que pour certain type d'états d'interface, la fréquence de mesure $1 \mathrm{MHz}$ soit insuffisante pour geler les pièges en régime de désertion, voire en régime d'inversion. Concernant le GaAs, Meiners [53] a pu observer à partir de mesures $C(V)$ à $150 \mathrm{MHz}$, qu'une densité significative de pièges en surface était en mesure de suivre les variations du signal de mesure.

Malgré ces inconvénients, cette méthode est cependant toujours utilisée pour l'évaluation des densités d'états d'interface pour différentes configurations MIS [54].

Cependant, les spectres obtenus par cette méthode doivent être considérés avec prudence.

Pour la structure a-GaAsN/c-GaAs(n) considérée, la distribution énergétique de ces états dans la bande interdite de GaAs est représentée sur la figure 6.

L'étude est limitée au régime de désertion. Les temps caractéristiques des pièges varient exponentiellement avec le potentiel de surface et les états proches du niveau de Fermi sont très rapides. Ils peuvent se charger et se décharger au rythme du signal test. Les points exclus de l'analyse ont été entourés de manière approximative d'un grisé.

Comme nous pouvons le constater, la densité d'état de surface est de l'ordre de $10^{11} \mathrm{~cm}^{-2} \mathrm{eV}^{-1}$ 


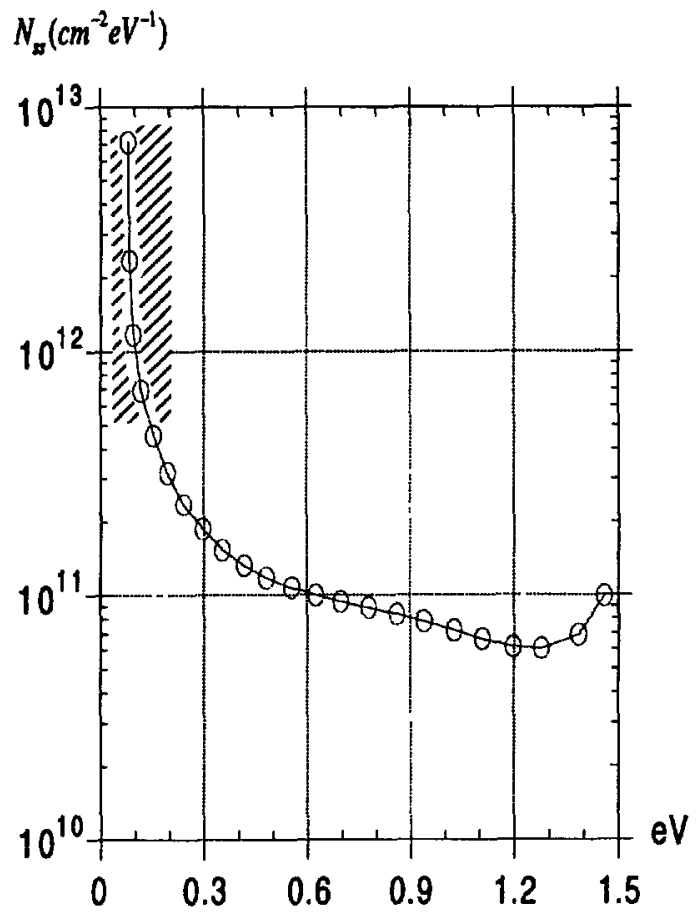

Fig. 6. - Distribution de la densité des états de surface à l'interface a-GaAsN/c-GaAs(n). [Density of surface states distribution at the a-GaAsN/c-GaAs(n) interface.]

au milieu du gap. Cette valeur est comparable à celles données dans la littérature concernant différents type de diélectriques $[44,54]$ et calculées par la même méthode. Ce résultat indique que l'interface entre a-GaAsN et $\mathrm{GaAs}(\mathrm{n})$ est de bonne qualité.

\section{Conclusion}

En pulvérisant de l'arséniure de gallium en atmosphère neutre d'argon, ou dans un mélange réactif d'argon et d'azote, sur des substrats monocristallins d'arséniure de gallium de type n, nous avons réalisé des structures a-GaAs/c-GaAs et a-GaAsN/c-GaAs dans le but de tester l'intérêt de ces matériaux comme couche de passivation de GaAs.

Les jonctions a-GaAs/c-GaAs obtenues à température ambiante, conduisent à des structures ayant une densité de défauts importante. L'interprétation des caractéristiques électriques est impossible par l'approximation des jonctions abruptes sans états d'interface. La résistivité du a-GaAs $\left(10^{7} \Omega \mathrm{cm}\right)$ mesurée à température ambiante s'est avérée être trop faible pour le but recherché.

Des couches minces de a-GaAsN ont été déposées par pulvérisation réactive dans un mélange d'argon et d'azote et à une température de $150^{\circ} \mathrm{C}$. Ces couches plus résistives permettent d'obtenir des structures qui présentent un comportement de type capacité MIS.

Pour ces structures, les caractéristiques $I(V)$ sont quasi-symétriques avec des densités de cou- 
rant plus faibles de trois ordres de grandeur environ que celles des structures a-GaAs/c-GaAs.

Nous avons établi l'existence d'une charge positive fixe dans la couche de a-GaAsN. La répartition des états d'interface déterminée par la méthode de Terman est de $10^{11} \mathrm{~cm}^{-2} \mathrm{eV}^{-1}$ au milieu de la bande interdite. Ce résultat prouve que l'interface est de bonne qualité. On peut espérer améliorer le dispositif en augmentant la résistivité du diélectrique utilisé.

\section{Bibliographie}

[1] Chittik R.C., Properties of glow discharge deposited amorphous Ge and Si, J. Non Cryst. Soluds 3 (1970) 255.

[2] Spear W.E. et Le Comber P.G., Substitutional doping of amorphous Si, Sol. State. Comm 17 (1975) 1193.

[3] Spear W.E. et Le Comber P.G., Electronic properties of substitutionally doped a-Si and a-Ge, Phil. Mag 33 (1976) 935.

[4] Snell A.J., Mackenzie K.D., LeComber P.G. et Spear W.E., The interpretation of capacitance and conductance measurements on metal-amorphous silicon barriers, Phil. Mag. B 40 (1979) 1.

[5] Beyer W. et Stuke J., Thermoelectric power of amorphous semiconductors, J. Non Cryst. Solids 8 (1972) 321.

[6] Hauser J.J., Disalvo F.J., Hutton J.R. et Hutton R.S., Localized gap states in amorphous semiconducting compounds, Phil. Mag. 135 (1977) 1557.

[7] Paul W., Moustakas T.D., Anderson D.A. et Freeman P., Proc. of 7th International Conf. Amorphous Semiconductors (Edinburgh, 1977) p. 467.

[8] Gheorghiu A. et Theye M.L., Relation between electronic properties and atomic structure of GaAs films, Proc. of 7th International Conf. Amorphous Semiconductors (Edinburgh, 1977) p. 462

[9] Schiller C., Gheorghiu A. et Theye M.L., Etude de la composition de couches minces de GaAs amorphe par analyse non dispersive de rayons X, J. Microsc. Spectrosc. Electron. 3 (1978) 255.

[10] Theye M.L., Gheorghiu A. et Launois M., Investigations of disorder effects in amorphous GaAs and GaP by EXAFS, J. Phys.-C : Sol. State Physics 13 (1980) 6569.

[11] Gheorghiu A. et Theye M.L., Disorder effects and the optical properties of amorphous GaAs and GaP, Phil. Mag.-B 44 (1981) 285.

[12] Gheorghiu A., Rappeneau T., Fisson S. et Theye M.L., Characterization of flash-evaporated amorphous GaAs, GaP and GaSb films as a function of deposition conditions, Thin Solid Films 120 (1984) 191.

[13] Senemaud C., Belin E., Gheorghiu A. et Theye M.L., XPS studies of disorder effects in flashevaporated amorphous GaAs, Sol. State Comm. 55 (1985) 947.

[14] Theye M.L. et Gheorghiu A., Comparative study of amorphous ge and amorphous GaAs, Phul. Mag.-B 52 (1985) 325.

[15] Theye M.L., Gheorghiu A., Rappeneau T. et Udron D., Electrical conductivity of nonhydrogenated amorphous tetracoordinated semiconductors, Disordered Semiconductors (Plenium Press, 1987) p. 489.

[16] Despax B., Aguir K. et Segui Y,, Procédé de dépôt d'au moins une couche d'un composé III-V ou II-VI en particulier GaAs et substrat revêtu d'un tel dépôt, Brevet Français $\mathbf{n}^{\circ}$ 85.06026, (Avril 1985).

[17] Despax B., Aguir K. et Segui Y., New plasma deposition process of a-Ga $\mathrm{As}_{1-x}$, Thin. Solid. Films 145 (1986) 233.

[18] Aguir K., Despax B., Hadidou H., Bandet J., Frandon J. et Bacquet G., Characterization of hydrogenated amorphous $\mathrm{Ga}_{x} \mathrm{As}_{1-x}$ thin films, Phil. Mag.-B 58 (1988) 6. 
[19] Aguir K., Hadidou H., Lauque P. et Despax B., Electrical and optical properties of R.F. glow discharge amorphous $\mathrm{Ga}_{x} \mathrm{As}_{1-x}$ films, J. Non Cryst. Solvds 113 (1989) 231.

[20] Alimoussa L., Carchano H. et Thomas J.P., Influence of hydrogen partial pressure on deposition and properties of sputtered amorphous GaAs, J. Phys. France C4 42 (1981) 683.

[21] Carchano H., Alimoussa L., Thomas J.P. et Fallavier M., Ion beam analysis techniques and characterization of a-GaAs:H, Nucl. Inst. Methods 218 (1983) 579.

[22] Carchano H., Alimoussa L., Fassi-Fihri A., Lalande F. et Loussier R., Etude de la structure cristalline et de la composition de films de GaAs pulvérisé, avec ou sans recuit laser, J. Phys. France C1-43 (1982) 341

[23] Carchano H., Lalande F. et Loussier R., Etude de la stoechiométrie de couches minces polycristallines de GaAs déposées par pulvérisation cathodique R.F., Thrn. Solvd. Films 120 (1984) 47.

[24] Carchano H., Lalande F. et Loussier R., Dépôt de couches minces d'AsGa polycristallin par pulvérisation cathodique R.F., Thin. Solid. Frlms 135 (1985) 107.

[25] Deville A., Gaillard B., Carchano H., Seedek K. et Stevens H., ESR in RF sputtered amorphous GaAs. The As+Ga, J. Phys. Cond. Matter 1 (1989) 9369.

[26] Carchano H., Seedek K. et Loussier R., Inpurity incorporation in a-GaAs, Le Vide, Les Couches Minces 235 (1987) 211.

[27] Carchano H., Seedek K. et Seguin J.L., Amelioration of electronic properties of a-GaAs thin films with view of realization of solar cells, Proc. of the Euroforum - New Energies Congress Saarbrucken(FRG) Vol. 3 (24-28 october, 1988).

[28] Aguir K., Fennouh A., Carchano H., Seguin J.L., El Hadadi B. et Lalande F., Electrical properties of a-GaAs/c-Si (p) heterojunctions, Thin Solvd Fulms 257 (1995) 98-103.

[29] Fennouh A., Aguir K., Carchano H., El Hadadi B. et Seguin J.L., Electrical characteristics of amorphous GaAs/n crystalline Si heterojunctions, accepté pour publication dans J. Mat. Sci. Eng. $B$ (1995).

[30] Fennouh A., Réalisation et caractérisation d'hétérojonctions a-GaAs/c-Si et a-GaAs/c-GaAs obtenues par pulvérisation cathodique RF, Thèse de Doctorat en Sciences (Univ. Aix-Marseille 3, 1993).

[31] Becke H.W., Hall R. et White J., Gallium arsenide MOS transistors, Solid-State Electronics, Vol. 8 (1965) p. 813-823.

[32] Becke H.W. et White J., Gallium arsenide FET'S outperform conventional silicon MOS devices, Electron. 40 (1967) 82.

[33] Logan R.A., Schwartz B. et Sandburg W.J., Anodic oxidation of Gallium arsenide in aqueous hydrogen peroxide solution, J. Electrochem. Soc. 120 (1973) 1385.

[34] Hasegawa H., Forward K.W. et Hartnagel H.L., New anodic native oxide of GaAs with improved dielectric and interface properties, Appl. Phys. Lett. 26 (1975) 567-569.

[35] Chang R.P.H., Some properties pf plasma-grown GaAs oxides, Thın. Solid. Films. 56 (1979) 89-106.

[36] Yamasaki K. et Sugano T., Anodic oxidation of Gallium arsenide using oxygen plasma, Jpn. $J$. Appl. Phys. 17 (1978) 321.

[37] Yokoyama N., Mimura T., Odani K. et Fukuta M., Low-temperature plasma oxidation of GaAs, Appl. Phys. Lett. 32 (1978) 58-60

[38] Thurmond C.D., Schwartz G.P., Kammlott G.W. et Schwartz B., GaAs oxydation and the GaAsoxygen equilibrium phase diagram, J. Electrochem. Sac. 127 (1980) 1366.

[39] Capasso F. et Williams G.F., A proposed hydrogenation/nitridization passivation mechanism for GaAs and other III-V semiconductor devices, including InGaAs long wavelenth photodetectors, $J$. Electrochem. Soc. 129 (1982) 821.

[40] Offsey S.D., Woodall J.M., Warren A.C., Kirchner P.D., Chappell T.I. et Pettit G.D., Unpinned (100) GaAs surfaces in air using photochemistry, Appl. Phys. Lett. 48 (1986) 475. 
[41] Taillepied N. et Gourrier S., Current drift mechanism in $\operatorname{In}_{0,53} \mathrm{Ga}_{0,47}$ As depletion mode metalinsulator field-effect transistors, Appl. Phys. Lett. 48 (1986) 978.

[42] Callegari A., Hoh P.D., Buchanan D.A. et Lacey D., Unpinned gallium oxide/GaAs interface by hydrogen and nitrogen surface plasma treatment, Appl. Phys. Lett. 54 (1989) 532.

[43] Callegari A., Sadana D.K., Buchanan D.A., Paccagnella A., Marshall E.D., Tischler M.A. et Norcott M.H., Properties of Si/GaAs structures formed by solid phase epitaxy of a-Si on GaAs, Appl. Phys. Lett. 58 (1991) 2540.

[44] Mui D.S.L., Biswas D., Reed J., Demirel A.L., Strite S. et Morkoç H., Investigations of the $\mathrm{Si}_{3} \mathrm{~N}_{4} / \mathrm{Si} / \mathrm{GaAs}$ insulator-semiconductor interface with low interface trap density, Apl. Phys. Lett. 60 (1992) 2511.

[45] Kumabe K. et Matsumoto N., Properties of GaAs and InP Amorphous-film/Crystal junctions, Jpn. J. Appl. Phys. 18 (1979) 1789.

[46] Wayers M., Sato M. et Ando H., Red shift of photoluminescence and absorption in dilute GaAsN alloy layers, Jpn. J. Appl. Phys. 31 (1992) L853.

[47] Wayers M. et Sato M., Growth of GaAsN alloys by low-pressure metalorganic chemical vapor deposition using plasma-cracked $\mathrm{NH}_{3}$, Appl. Phys. Lett. 62 (1993) 1396.

[48] van Gelder W. et Nicollian E.H., J. Electrochem. Soc. 118 (1971) 138.

[49] Grove A.S., Physique et technologie des dispositifs à semiconducteur (Dunod, Paris, 1971).

[50] Nicollian E.H. et Brews J.R., MOS Physics and Technology (Wiley, New York, 1982).

[51] Sze S.M., Physics of Semiconductor Devices, 2nd Edition (John Wiley \& Sons, New York, 1981).

[52] Terman L.M., An investigation of surface states at a Silicon/Silicon oxide interface employing Metal-Oxide-Silicon diodes, Solzd-State. Electron. 5 (1962) 285.

[53] Meiners L.G., Electrical properties of the gallium arsenide-insulator interface, J. Vac. Sci. Technol. 15 (1978) 1402-1407.

[54] Herman J.S. et Terry F.L., Hydogen sulfide plasma passivation of gallium arsenide, Appl. Phys. Lett. 60 (1992) 716. 